\title{
Time-Reversal Symmetry Breaking Driven Topological Phase Transition in EuB $\mathbf{B}_{6}$
}

\author{
Shun-Ye Gao $\odot,{ }^{1,2, *}$ Sheng Xu, ${ }^{3,4, *}$ Hang Li, ${ }^{5,6, *}$ Chang-Jiang Yi®, ${ }^{1, *}$ Si-Min Nie, ${ }^{7}$ Zhi-Cheng Rao $\odot,{ }^{1,2}$ Huan Wang, ${ }^{3,4}$ \\ Quan-Xin Hu, ${ }^{1,2}$ Xue-Zhi Chen, ${ }^{2,8}$ Wen-Hui Fan, ${ }^{1,2}$ Jie-Rui Huang $\odot,{ }^{1,2}$ Yao-Bo Huang, ${ }^{9}$ Nini Pryds, ${ }^{5}$ Ming Shi, ${ }^{6}$ \\ Zhi-Jun Wang $\odot^{1,2}$ You-Guo Shi, ${ }^{1,2,10,8}$ Tian-Long Xia, ${ }^{3,4,}$ Tian Qian $\odot,{ }^{1,10, \dagger}$ and Hong Ding ${ }^{1,2,10,11}$ \\ ${ }^{1}$ Beijing National Laboratory for Condensed Matter Physics and Institute of Physics, \\ Chinese Academy of Sciences, Beijing 100190, China \\ ${ }^{2}$ University of Chinese Academy of Sciences, Beijing 100049, China \\ ${ }^{3}$ Department of physics, Renmin University of China, Beijing 100872, China \\ ${ }^{4}$ Beijing Key Laboratory of Opto-electronic Functional Materials \& Micro-nano Devices, \\ Renmin University of China, Beijing 100872, China \\ ${ }^{5}$ Department of Energy Conversion and Storage, Technical University of Denmark, \\ Fysikvej Building 310, DK-2800 Kgs. Lyngby, Denmark \\ ${ }^{6}$ Swiss Light Source, Paul Scherrer Institut, CH-5232 Villigen, Switzerland \\ ${ }^{7}$ Department of Materials Science and Engineering, Stanford University, Stanford, California 94305, USA \\ ${ }^{8}$ Shanghai Institute of Applied Physics, Chinese Academy of Sciences, Shanghai 201800, China \\ ${ }^{9}$ Shanghai Synchrotron Radiation Facility, Shanghai Advanced Research Institute, \\ Chinese Academy of Sciences, Shanghai 201204, China \\ ${ }^{10}$ Songshan Lake Materials Laboratory, Dongguan, Guangdong 523808, China \\ ${ }^{11}$ CAS Center for Excellence in Topological Quantum Computation, \\ University of Chinese Academy of Sciences, Beijing 100049, China
}

(Received 18 November 2020; revised 25 January 2021; accepted 24 February 2021; published 19 April 2021)

The interplay between time-reversal symmetry (TRS) and band topology plays a crucial role in topological states of quantum matter. In time-reversal-invariant (TRI) systems, the inversion of spindegenerate bands with opposite parity leads to nontrivial topological states, such as topological insulators and Dirac semimetals. When the TRS is broken, the exchange field induces spin splitting of the bands. The inversion of a pair of spin-splitting subbands can generate more exotic topological states, such as quantum anomalous Hall insulators and magnetic Weyl semimetals. So far, such topological phase transitions driven by the TRS breaking have not been visualized. In this work, using angle-resolved photoemission spectroscopy, we have demonstrated that the TRS breaking induces a band inversion of a pair of spinsplitting subbands at the TRI points of Brillouin zone in $\mathrm{EuB}_{6}$, when a long-range ferromagnetic order is developed. The dramatic changes in the electronic structure result in a topological phase transition from a TRI ordinary insulator state to a TRS-broken topological semimetal (TSM) state. Remarkably, the magnetic TSM state has an ideal electronic structure, in which the band crossings are located at the Fermi level without any interference from other bands. Our findings not only reveal the topological phase transition driven by the TRS breaking, but also provide an excellent platform to explore novel physical behavior in the magnetic topological states of quantum matter.

DOI: $10.1103 /$ PhysRevX.11.021016

\footnotetext{
${ }^{*}$ These authors contributed equally to this work.

Corresponding author. tqian@iphy.ac.cn

Corresponding author. tlxia@ruc.edu.cn

${ }^{\S}$ Corresponding author. ygshi@iphy.ac.cn
}

Published by the American Physical Society under the terms of the Creative Commons Attribution 4.0 International license. Further distribution of this work must maintain attribution to the author(s) and the published article's title, journal citation, and DOI.
Subject Areas: Condensed Matter Physics, Magnetism, Topological Insulators

\section{INTRODUCTION}

Since the discovery of the integer quantum Hall effect, time-reversal symmetry (TRS) has been a key issue in the study of topological states of quantum matter [1-3]. The thinking and research on TRS directly led to the discovery of time-reversal-invariant (TRI) topological insulators with the quantum spin Hall effect [4-9]. In magnetic materials, the TRS breaking induced by magnetic order can produce more exotic topological states. The most famous examples are the realization of quantum anomalous Hall (QAH) effect [10-19] and the discovery of magnetic Weyl 
semimetals [20-23]. The interplay of magnetism and band topology may lead to the emergence of novel physical behavior, which would give new access to applications of topological states of quantum matter.

The QAH insulator and magnetic Weyl semimetal states are closely related to the band inversion under the TRS breaking. As illustrated in Fig. 1(a), regardless of whether the initial TRI systems with inversion symmetry are topological insulators with band inversion or ordinary insulators, when the TRS is broken, the exchange field leads to an inversion of one pair of spin-splitting subbands with opposite chirality at the TRI point, whereas the other pair is separated. In two-dimensional (2D) systems, the band inversion opens an energy gap characterized by a nonzero Chern number, forming the QAH insulators [10]. In three-dimensional systems, the band inversion cannot be
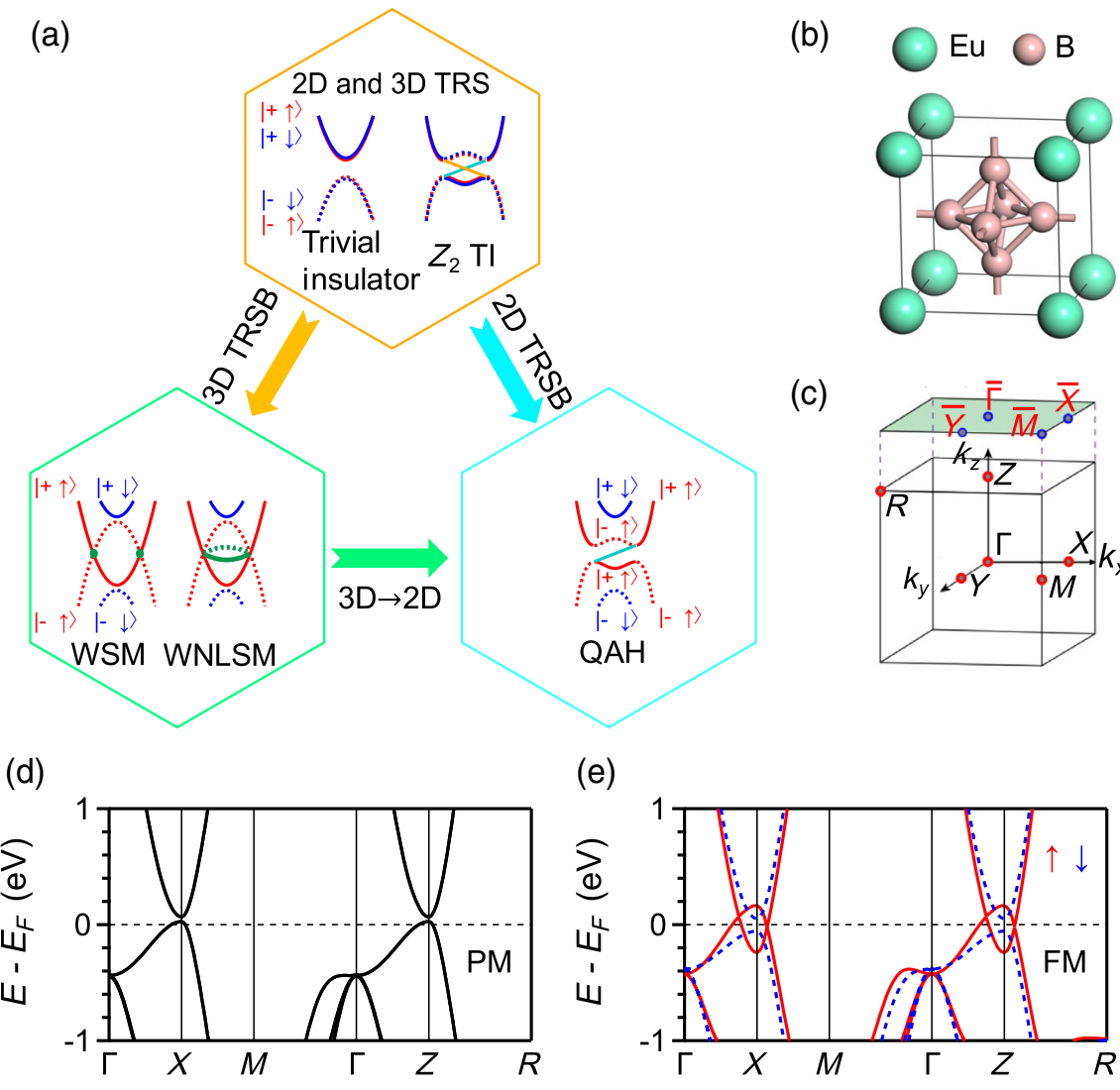

(c)

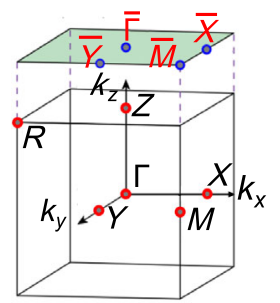

(e)
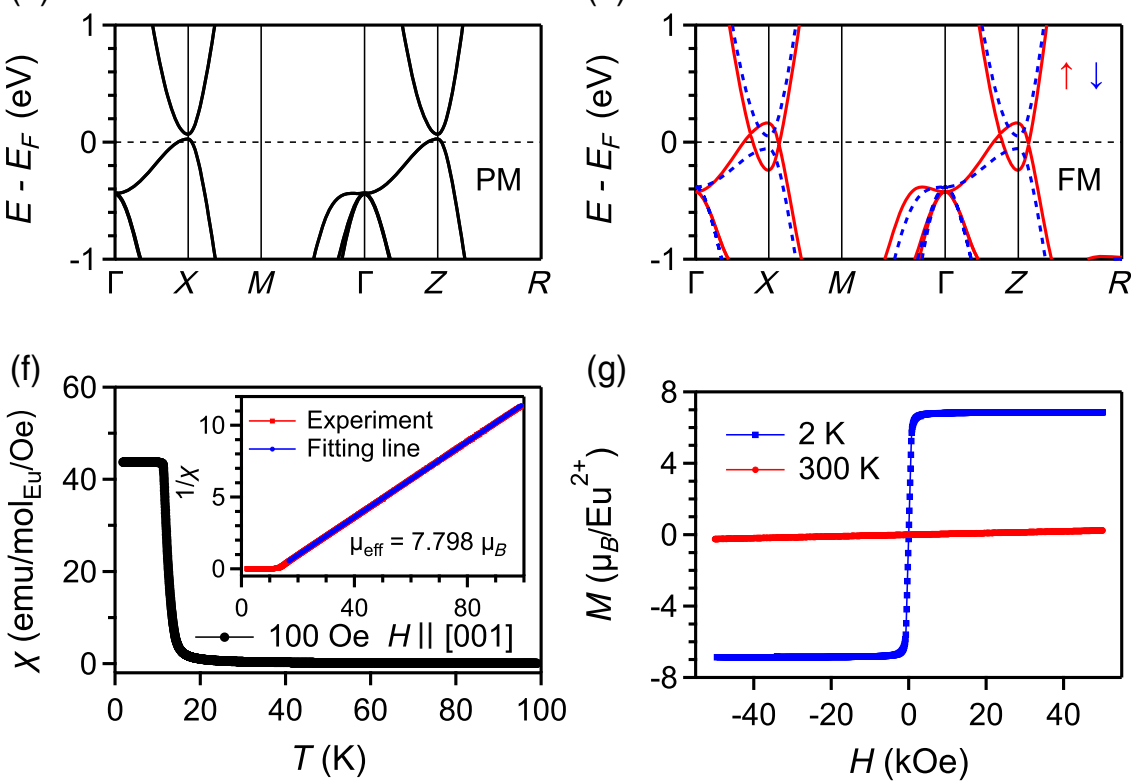

FIG. 1. Calculated electronic structures and magnetic properties of EuB 6 . (a) Schematic plots of emergent topological phases with TRS breaking (TRSB) in centrosymmetric 3D and 2D systems. Topological insulator (TI), Weyl semimetal (WSM), and Weyl nodal-line semimetal (WNLSM). "+" and "-" represent opposite parity of the subbands. "个” and " $\downarrow$ " represent spin up and spin down. (b) Crystal structure of $\mathrm{EuB}_{6}$. (c) Bulk BZ and (001) surface BZ. (d),(e) Calculated electronic structures along high-symmetry lines in the PM and FM states, respectively. Magnetic moments are defined to be along the [001] direction in the FM state. The calculated results are adopted from Ref. [24]. (f) Temperature dependence of magnetic susceptibility. Inset: temperature dependence of inverse susceptibility. The blue line is a fitting to the Curie-Weiss formula. (g) Magnetic field dependence of magnetization at $2 \mathrm{~K}$ (blue) and $300 \mathrm{~K}$ (red) with magnetic fields parallel to the [001] direction. 
fully gapped even with spin-orbit coupling, forming the magnetic topological semimetals (TSMs) with twofold degenerate Weyl points or nodal lines [24]. Under the 2D limit, the degeneracy is lifted by opening an energy gap and the magnetic TSMs are transformed into the QAH insulators $[20,24]$.

While it is believed that the QAH insulator state in magnetically doped $\mathrm{Bi}_{2} \mathrm{Te}_{3}$ [10-15] and $\mathrm{MnBi}_{2} \mathrm{Te}_{4}$ [16-19] and the magnetic Weyl semimetal state in $\mathrm{Co}_{3} \mathrm{Sn}_{2} \mathrm{~S}_{2}$ [20-23] arise from the mechanism illustrated in Fig. 1(a), the topological phase transitions driven by the TRS breaking have not been observed. In this work, we use angle-resolved photoemission spectroscopy (ARPES) to visualize the topological phase transition from a TRI ordinary insulator state to a TRS-broken TSM state in $\mathrm{EuB}_{6}$, which is accompanied by the development of a longrange ferromagnetic (FM) order. Remarkably, the band crossings are the only feature at the Fermi level $\left(E_{F}\right)$ without the existence of other Fermi surfaces (FSs), which is favorable to the emergence of exotic physics associated with the topological nodes.

\section{METHODS}

Single crystals of $\mathrm{EuB}_{6}$ were grown by the flux method. The ingredients with a ratio of $\mathrm{Eu}: \mathrm{B}: \mathrm{Al}=1: 6: 570$ were put into the alumina crucible with a cap and sealed into a quartz ampoule. Then the ampoule was heated to $1350^{\circ} \mathrm{C}$, kept for $10 \mathrm{~h}$, and cooled to $700^{\circ} \mathrm{C}$ at a rate of $5^{\circ} \mathrm{C}$ per hour. $\mathrm{EuB}_{6}$ single crystals were obtained after the $\mathrm{Al}$ flux was removed by dissolving in sodium hydroxide solution. All the ARPES data shown were collected at the "dreamline" beam line and the 03U beam line at the Shanghai Synchrotron Radiation Facility, the SIS-HRPES beam line at the Swiss Light Source, and the BL25SU beam line at the Spring 8 . We conducted complementary experiments at the $13 \mathrm{U}$ beam line at the National Synchrotron Radiation Laboratory at Hefei, the "Bloch" beam line, MAXIV, Sweden, the 7.0.2.1 beam line, ALS, U.S., and the I05 beam line, Diamond, UK. The energy and angular resolutions were set to $15-70 \mathrm{meV}$ and $0.1^{\circ}$. The samples were cleaved in situ and measured in ultrahigh vacuum better than $7 \times 10^{-11}$ torr.

\section{RESULTS}

$\mathrm{EuB}_{6}$ has the CsCl-type crystal structure with space group Pm3m (no. 221) [25]. As seen in Fig. 1(b), Eu atoms are located at the corners of cubic lattice and six B atoms form an octahedral cage inside the lattice. The magnetic susceptibility $(\chi)$ curves in Fig. $1(f)$ show that $E B_{6}$ is in the paramagnetic (PM) state at high temperatures. The fitting to the Curie-Weiss formula gives an effective magnetic moment $\mu_{\text {eff }}=7.798 \mu_{B} / \mathrm{Eu}^{2+}$, which is close to the theoretical value $7.94 \mu_{B}$ of local $4 f^{7}$ orbitals. The magnetic susceptibility starts to rise sharply at $\sim 15 \mathrm{~K}$ and reaches a plateau at $\sim 11 \mathrm{~K}$ in Fig. 1(f), indicating a PM to FM transition consistent with previous reports [26-28]. Specific heat measurements revealed two consecutive phase transitions at $T_{M}=15.5 \mathrm{~K}$ and $T_{C}=12.6 \mathrm{~K}$ [29], which were subsequently confirmed and extensively studied by various experimental techniques [30-33]. These studies indicate that isolated magnetic polarons start to form at $\sim 30 \mathrm{~K}$, and they grow in size and/or increase in number with decreasing temperature. The magnetic polarons overlap and percolate in the bulk at $T_{M}$, forming a ferromagnetically ordered phase coexisting with the PM phase. With further decreasing temperature, the volume fraction of the FM phase increases at the expense of the PM phase, and eventually a complete FM state is achieved at $T_{C}$. The adiabatic magnetization curve at $2 \mathrm{~K}$ in Fig. $1(\mathrm{~g})$ shows that $\mathrm{EuB}_{6}$ is a soft ferromagnet with an extremely low coercive field $\sim 35$ Oe.

Theoretical calculations indicate that $\mathrm{EuB}_{6}$ is a semiconductor with trivial topology in the PM state [24]. Both valence band top and conduction band bottom are located at three TRI points, $X, Y$, and $Z$, which are equivalent in the PM state, forming a direct band gap of $\sim 20 \mathrm{meV}$ at $E_{F}$ in Fig. 1(d). The valence and conduction bands near $E_{F}$ are mainly from the $\mathrm{B} 2 p$ and $\mathrm{Eu} 5 d$ states, respectively. When the $\mathrm{Eu}^{2+} 4 f$ magnetic moments form a $\mathrm{FM}$ order, the interatomic antiferromagnetic exchange coupling between the B $2 p$ and occupied (unoccupied) Eu $4 f$ states pushes the spin-up (spin-down) valence subband upward (downward), while the intra-atomic FM exchange coupling between the Eu $5 d$ and occupied (unoccupied) $4 f$ states pushes the spin-up (spin-down) conduction subbands downward (upward) $[24,34,35]$. Because of the special exchange coupling, the band inversion occurs at three TRI points in the spin-up channel, while the band gap increases in the spin-down channel, as seen in Fig. 1(e). According to theoretical analysis [24], the band inversion at these TRI points cannot be fully gapped, forming Weyl points or nodal lines with twofold degeneracy, depending on the magnetic moment direction. When the magnetic moments are aligned with the [001] direction, the mirror symmetry $\hat{M}_{z}$ protects three nodal lines encircling three TRI points, respectively. When the magnetic moments are aligned with the [110] direction, the mirror symmetry $\hat{M}_{110}$ protects one nodal line encircling the $Z$ point while Weyl points can be found near the $X$ and $Y$ points. When the magnetic moments deviate from the [001] and [110] directions, no nodal lines survive because the mirror symmetries are broken, but Weyl points always exist near three TRI points and their positions move with varying the magnetic moment direction.

We first measure electronic structures in the PM state $(T=20 \mathrm{~K})$ on $(001)$ cleavage surfaces of $\mathrm{EuB}_{6}$ with ARPES. We observe two sets of distinct electronic structures near $E_{F}$, and the corresponding core level spectra have different peak structures. As seen in Fig. 2(a), the 
(a)

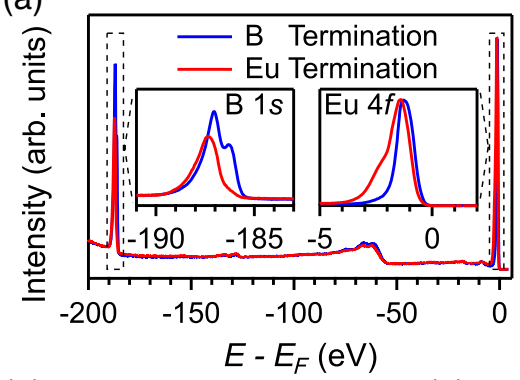

(d)

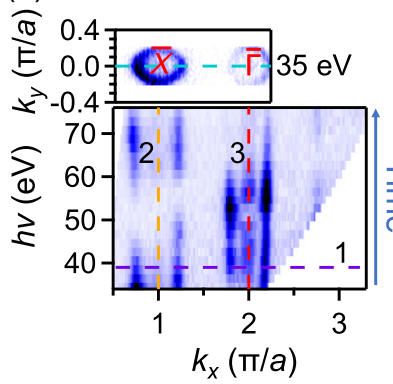

(g)

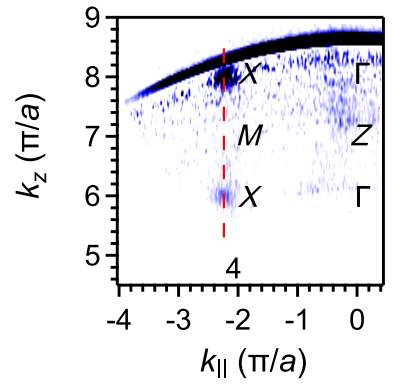

(b)

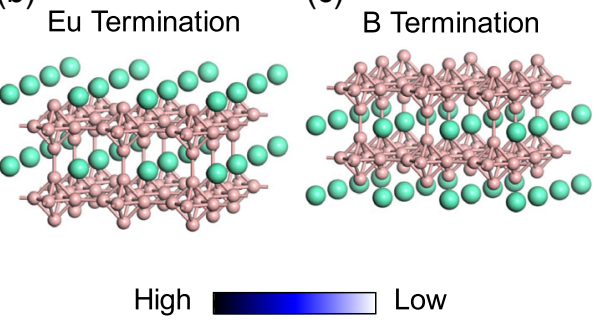

(e)

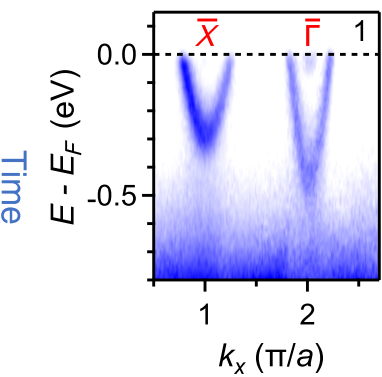

(h)

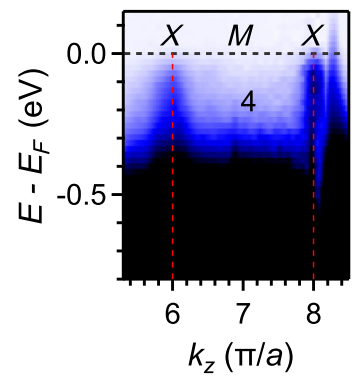

(f)

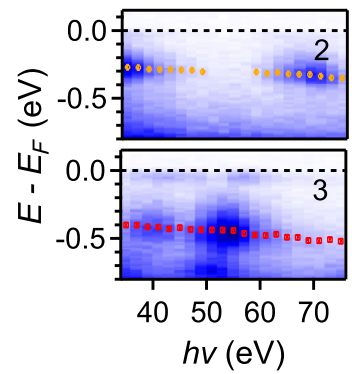

(i)

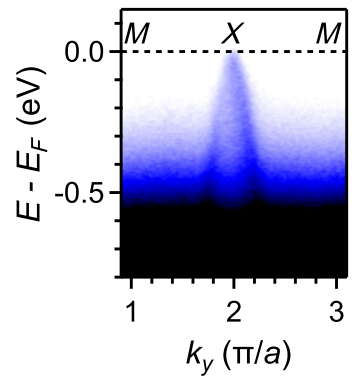

FIG. 2. Electronic structures measured on Eu- and B-terminated surfaces in the PM state. (a) Core-level photoemission spectra showing characteristic peaks of the B $1 s$ and Eu $4 f$ orbitals in the insets. (b),(c) Schematic plots of (001) cleavage surfaces with Eu and B terminations, respectively. (d) Top: intensity plot of the ARPES data at $E_{F}$ with $h v=35 \mathrm{eV}$. Bottom: intensity plot of the ARPES data at $E_{F}$ collected in a range of photon energies from 34 to $76 \mathrm{eV}$. (e) Intensity plot of the ARPES data along cut 1 . (f) Intensity plots of the ARPES data along cuts 2 and 3, showing photon energy and time dependence of the band bottoms. (g) Intensity plot of the ARPES data at $E_{F}$ collected along the direction from $(0,0)$ to $(-\pi / a, 2 \pi / a)$ in a range of photon energies from 59 to $151 \mathrm{eV}$. The inner potential $V_{0}=17.73 \mathrm{eV}$ and the lattice constant $a=4.187 \AA$. (h) Intensity plot of the ARPES data along cut 4. (i) Intensity plot of the ARPES data along $M-X$ in the $k_{x}-k_{y}$ plane with $k_{z}=8 \pi / a$. The data in (d)-(f) and (g)-(i) were collected on Eu- and B-terminated surfaces, respectively. All the data were collected at $20 \mathrm{~K}$.

B $1 s$ (Eu $4 f$ ) states have single-peak (double-peak) and double-peak (single-peak) structures, respectively. As chemical environments of the atoms in the outmost layer are different from those of the atoms underneath, the atoms in the outmost layer can produce additional peaks in the core level states. We attribute the double-peak structures of the B $1 s$ and $\mathrm{Eu} 4 f$ states to two kinds of terminated surfaces with either B or Eu atoms in the outmost layer, as illustrated in Figs. 2(b) and 2(c). Recent scanning tunnelling microscopy measurements on (001) cleavage surfaces of $\mathrm{EuB}_{6}$ found two kinds of distinctive topographies, which were assigned to Eu- and B-terminated surfaces, respectively [36,37]. By scanning the Eu $4 f$ and B $1 s$ core levels at different positions on the cleavage surfaces with incident light, whose spot size varies between $30 \times 50$ and $50 \times 150 \mu \mathrm{m}^{2}$ at various synchrotron beam lines, we can find single termination areas with only one set of electronic structures. The results were repeated in independent measurements on eight samples.

The electronic structures measured on Eu-terminated surfaces are plotted in Figs. 2(d)-2(f). We observe two electron bands near $E_{F}$ at $\bar{\Gamma}$ and $\bar{X}$, respectively, where $a$ is the lattice constant. Figure 2(f) shows that the bottoms of both bands have no obvious dispersion with varying photon energy $(h v)$ except for the downward shift during measurements (see more details on time-dependent changes of the bands in Fig. 5 and 6 in the Appendix A). The nondispersive characteristic along the surfaceperpendicular momentum direction $k_{z}$ indicates that the bands observed on Eu-terminated surfaces are surface 
states. The electron band at the $\bar{X}$ point as well as its energy shift with time was also found in previous ARPES experiments [38,39], where it was speculated that the freshly cleaved surfaces could be metastable and relax with time, resulting in time-dependent band bending.

On the contrary, the $h v$-dependent data collected on B-terminated surfaces in Figs. 2(g) and 2(h) exhibit an obvious $k_{z}$ dispersion with a period of $2 \pi / a$, which is an indication of bulk states. We observe near- $E_{F}$ electronic states around the $X$ point, which show a hole band with top very close to $E_{F}$ in Fig. 2(i). Moreover, a shallow electron band just above the hole band is observed in some samples (see Fig. 7 in Appendix B). The observation is well consistent with the calculated bulk electronic structure of the PM state in Fig. 1(d), and it was not captured in previous ARPES experiments $[38,39]$. It is expected that the near- $E_{F}$ electronic states can be observed at $\bar{\Gamma}$ and $\bar{X}$ (or $\bar{Y})$, which are the (001)-surface projections of three TRI points. However, we find that the hole band is only clear at $(\pi / a, 2 \pi / a)$ on the second BZ boundary, while it is unclear or almost invisible at other locations, such as $(0,0)$, $(\pi / a, 0)$, and $(2 \pi / a, 0)$.

When the samples are cooled down from the PM to FM state, the surface states on Eu-terminated surfaces do not change except for the downward shift with time (Fig. 6 in Appendix A), whereas the near- $E_{F}$ electronic states on B-terminated surfaces exhibit dramatic changes. In Fig. 3(a) one can see clear band splitting with decreasing temperature through $T_{C}$ on B-terminated surfaces. After the temperature-dependent measurements, we heat up the sample to $17 \mathrm{~K}$ and confirm that the band dispersions can be restored to the initial state in the PM state, thus excluding the possibility of time-dependent changes. The band splitting is an indication of symmetry breaking, such as the breaking of TRS or inversion symmetry. The former induces the Zeeman splitting with energy shifts while the latter induces the Rashba splitting with momentum shifts. In $\mathrm{EuB}_{6}$, it is clear that the TRS is broken at low temperatures due to the long-range FM order, whereas X-ray and neutron diffraction experiments did not find any signature of a structural phase transition around $T_{C}[30,40]$, indicating that the inversion symmetry is preserved in the FM state. Therefore, we attribute the dramatic changes to the Zeeman splitting, which leads to energy shifts of the bands.

As seen in Fig. 3(b), there is a hole band $(\alpha)$ below $E_{F}$ at the $X$ point at $20 \mathrm{~K}$, and the band splits into two subbands $\left(\alpha_{1}\right.$ and $\left.\alpha_{2}\right)$ at $5 \mathrm{~K}$. The two subbands move up and down by $\sim 200 \mathrm{meV}$, respectively, from 20 to $5 \mathrm{~K}$. On the other hand, we observe that an electron band $\left(\beta_{1}\right)$ moves down with decreasing temperature. Because of the TRS breaking,
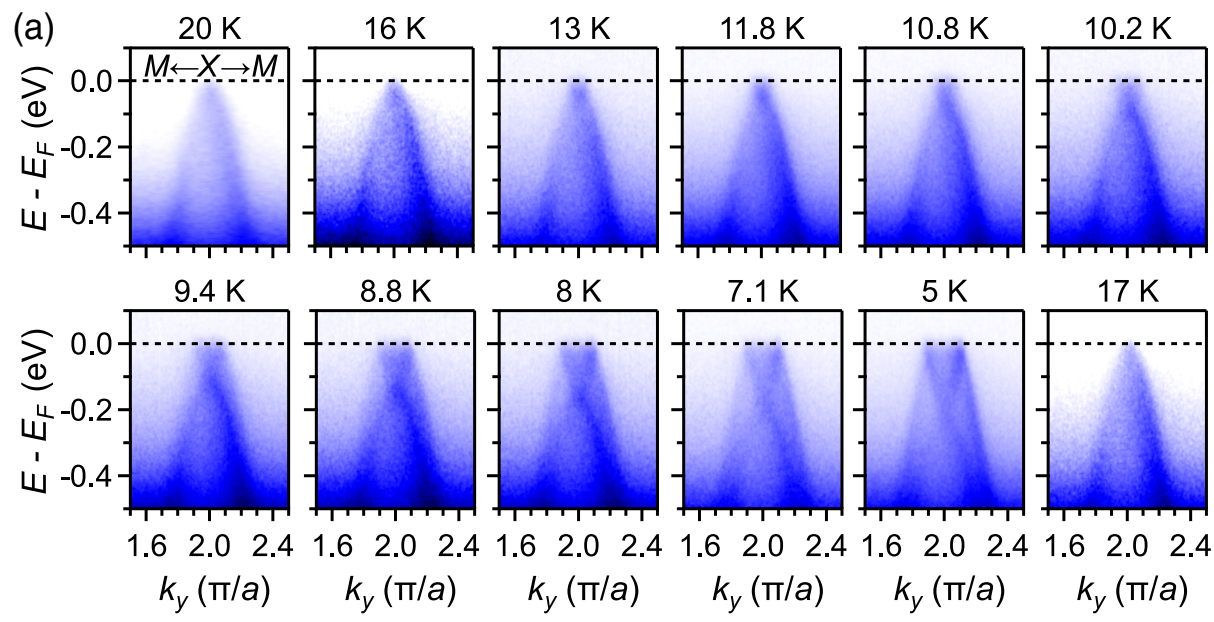

$k_{y}(\pi / a)$

$k_{y}(\pi / a)$

$k_{y}(\pi / a)$

$k_{y}(\pi / a)$

$k_{y}(\pi / a)$

(b)

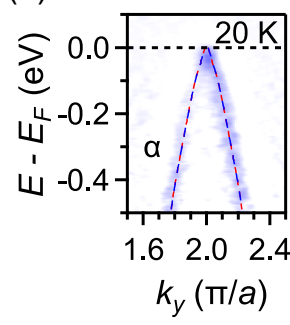

(c)

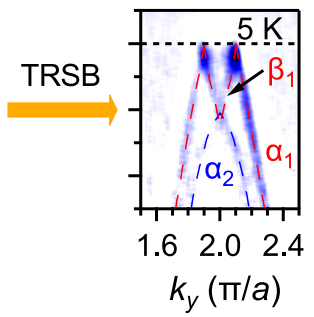

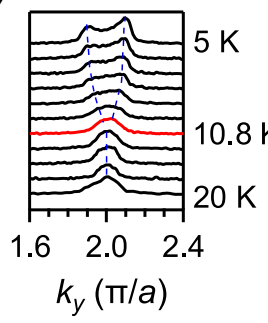

(d)

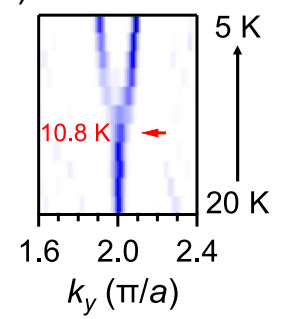

FIG. 3. Temperature dependence of band dispersions on B-terminated surfaces. (a) Intensity plots of the ARPES data along $M$ - $X$ at different temperatures between 20 and $5 \mathrm{~K}$. (b) $2 \mathrm{D}$ curvature intensity plots of the data in (a) at 20 and $5 \mathrm{~K}$. Dashed lines are guide to eyes of the band dispersions. (c) MDCs at $E_{F}$ of the ARPES data in (a). (d) Curvature intensity plot of the data in (c). All the data were collected with $h v=135 \mathrm{eV}$. 
the conduction band just above $E_{F}$ should also split into two subbands. One of them moves down across $E_{F}$, whereas the other should move up and therefore cannot be detected in the ARPES experiments. The dramatic changes in the electronic structure through $T_{C}$ are consistent with optical conductivity experiments, which revealed a substantial shift toward higher frequencies of the plasma edge below $T_{C}$, suggesting an increase of itinerant charge carriers and/or a reduction of their effective mass [29].

Notably, the band splitting results in the band inversion of two subbands $\alpha_{1}$ and $\beta_{1}$ at the $X$ point. The band (a)

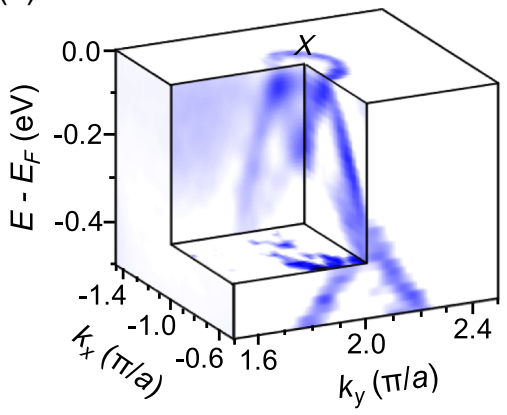

(b)

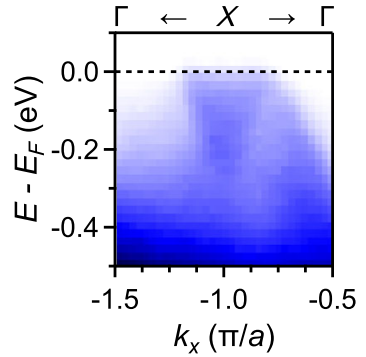

(c)

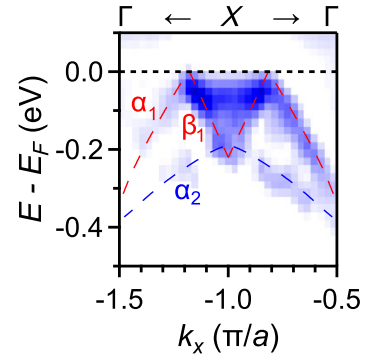

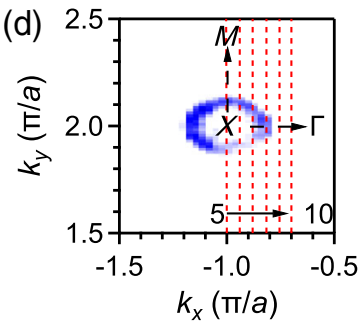

(e)
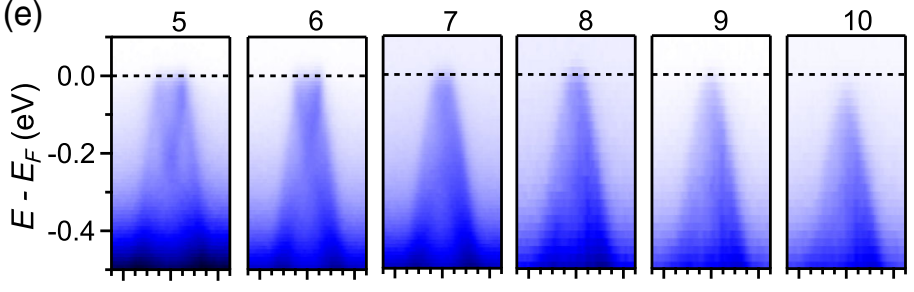

(g)
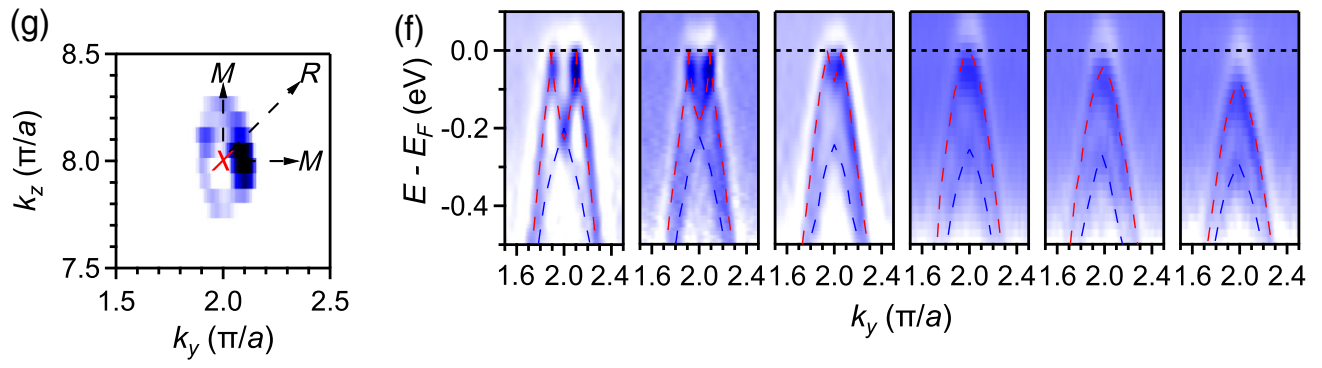

(h) $\quad 126 \mathrm{eV}$

(i)
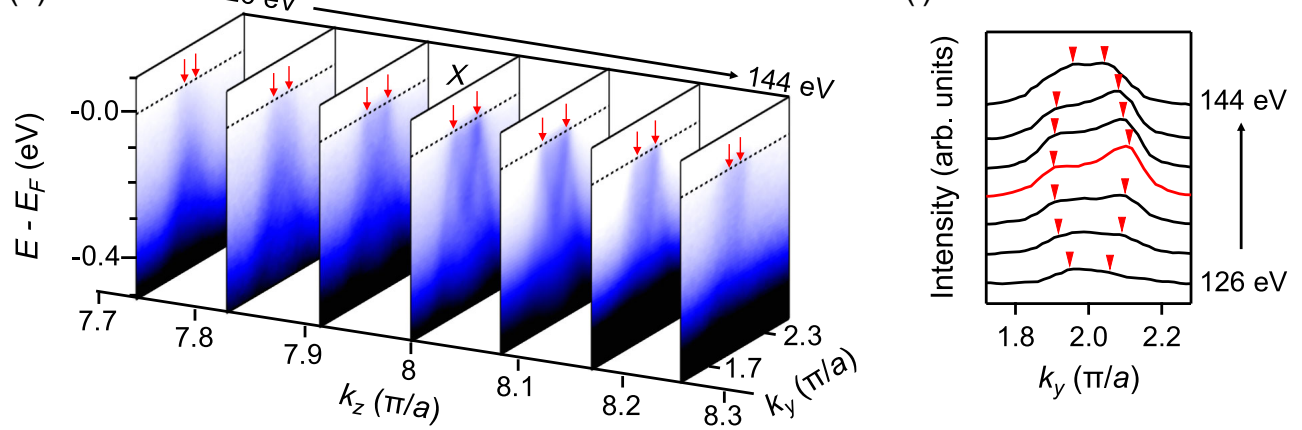

FIG. 4. In-plane and out-of-plane band dispersions on B-terminated surfaces in the FM state. (a) 3D curvature intensity plot of the ARPES data around the $X$ point. (b) Intensity plot of the ARPES data along $\Gamma-X$. (c) 2D curvature intensity plot of the data in (b). Dashed lines are guide to eyes of the band dispersions. (d) Curvature intensity plot of the ARPES data at $E_{F}$ around the $X$ point in the $k_{x}-k_{y}$ plane. (e) Intensity plots of the ARPES data along cuts 5-10. (f) Curvature intensity plots of the ARPES data in (e). (g) Curvature intensity plot of the ARPES data at $E_{F}$ around the $X$ point in the $k_{y}-k_{z}$ plane. (h) Intensity plots of the ARPES data along $M-X$ collected in a range of photon energies from 126 to $144 \mathrm{eV}$. (i) MDCs at $E_{F}$ of the ARPES data in (h). The data in (a)-(f) were collected with $h v=135 \mathrm{eV}$. All the data were collected at $4.5 \mathrm{~K}$. 
inversion agrees well with the calculations in the FM state in Fig. 1(e) [24]. Moreover, the calculations indicate that two subbands $\alpha_{2}$ and $\beta_{1}$ are also inverted in Fig. 1(e), while the experimental data show that they are almost degenerate at $\sim 0.2 \mathrm{eV}$ below $E_{F}$ in Fig. 3(b). In the experimental data both $\alpha_{2}$ and $\beta_{1}$ move down significantly, whereas in the calculations only $\beta_{1}$ moves down significantly while $\alpha_{2}$ moves down slightly, suggesting that the exchange coupling between the $\mathrm{B} 2 p$ and unoccupied $\mathrm{Eu} 4 f$ bands is underestimated in the calculations [24]. Despite the discrepancy, both experiment and calculation consistently confirm the band inversion of $\alpha_{1}$ and $\beta_{1}$, which is critical for the magnetic TSM state in $\mathrm{EuB}_{6}$.

Figures 3(c) and 3(d) show temperature-dependent momentum distribution curves (MDCs) at $E_{F}$ from the PM to FM state. The MDCs exhibit a single peak at $20 \mathrm{~K}$, corresponding to the hole band top in the PM state. The peak starts to split at $\sim 11 \mathrm{~K}$, which can be regarded as an indication of the band inversion. We notice that the band dispersions are not well defined between 11 and $8 \mathrm{~K}$ in Fig. 3(a). The phenomenon is probably related to the phase separation between $T_{M}$ and $T_{C}$, where the FM phase formed by magnetic polarons coexists with the PM phase [29-33]. The band dispersions become clear below $8 \mathrm{~K}$, probably because the complete FM state is achieved. The temperature range between 11 and $8 \mathrm{~K}$ is lower than the two transition temperatures $T_{M}=15.5 \mathrm{~K}$ and $T_{C}=12.6 \mathrm{~K}$ determined by specific heat and magnetization measurements [29,30]. In our ARPES experiments, the actual sample temperature may be higher than that detected by the sensor because the samples are exposed to thermal radiation from the room-temperature chamber and analyzer.

We systematically investigate in-plane and out-of-plane band dispersions near the $X$ point in the FM state. The results in Figs. 4(a)-4(f) exhibit that the band crossings at $E_{F}$ form a nodal-ring FS encircling the $X$ point in the $k_{x}-k_{y}$ plane. As seen in Fig. 4(d), the FS is elliptical with its long axis along $\Gamma-X$. Similarly, the $h v$-dependent data in Figs. 4(g)-4(i) show a nodal-ring FS formed by the band crossings in the $k_{y}-k_{z}$ plane. The FS should be nearly isotropic with fourfold symmetry in the $M-X-R$ plane, while the experimental FS in Fig. 4(g) appears to be stretched along $k_{z}$, which is caused by momentum broadening in the direction perpendicular to the sample surface. The FS formed by the band crossings can be regarded as the degeneracy of hole and electron FSs. de Haas-van Alphen and Shubnikov-de Haas ( $\mathrm{SdH})$ experiments found both hole and electron FSs with different sizes in the FM state $[41,42]$, which could arise from the FS splitting when the chemical potential slightly deviates from the band crossings due to small nonstoichiometry. The extremal areas of the ellipsoidal FS in the ARPES data are estimated to be 278 and $410 \mathrm{~T}$ in the $X-M-R$ and $\Gamma-X-M$ planes, respectively, which are $\sim 15 \%$ smaller than those determined by the $\mathrm{SdH}$ experiments at $0.4 \mathrm{~K}$ [42]. As seen in Fig. 3(d), the Fermi wave vector continues to increase below $11 \mathrm{~K}$ and does not reach saturation at $5 \mathrm{~K}$. The discrepancy in FS size can be attributed to the temperature difference between ARPES and $\mathrm{SdH}$ experiments.

Finally, we discuss the effects of magnetic domains. As magnetic fields are not allowed in the ARPES experiments, magnetic domains must exist in the soft ferromagnet $\mathrm{EuB}_{6}$. Because of the small magnetic anisotropy in $\mathrm{EuB}_{6}$, the typical domain size at zero field was estimated to be on the order of $1 \mu \mathrm{m}$ [30], which is much smaller than the spot size of incident light, suggesting that the ARPES results should be a mixture of electronic structures of multiple domains with various magnetic moment directions. As the magnetic moment direction determines the band crossings at $E_{F}$, specific topological states cannot be distinguished in the mixed electronic structure of multiple domains. On the other hand, the effects of magnetic moment direction on the overall band dispersions are negligible [24]. Therefore, despite the existence of magnetic domains, clear band dispersions can be observed when the samples enter the complete FM state at low temperatures.

\section{OUTLOOK}

Our ARPES data have demonstrated that the TRS breaking results in a topological phase transition with the band inversion at the $X$ point in $\mathrm{EuB}_{6}$, which is well consistent with the band calculations [24]. According to theoretical analysis, the band inversion at the TRI point cannot be fully gapped, forming Weyl points or nodal lines depending on the magnetic moment direction. As a soft ferromagnet, it is easy to align the magnetic moments with external magnetic fields, which would be favorable to tune the topological states and relevant physical properties. Compared with the other experimentally discovered magnetic TSMs $[21,23,43]$, the distinguishing characteristic in $\mathrm{EuB}_{6}$ is that the band crossings are the only feature at $E_{F}$ without the existence of other FSs, and therefore their energy positions are restricted at $E_{F}$ by electron-hole compensation in stoichiometric samples.

Band calculations indicate that the band inversion occurs in the spin-up channel whereas the band gap remains in the spin-down channel, suggesting that $\mathrm{EuB}_{6}$ is also a halfmetal with a full spin polarization at $E_{F}$ in the FM state $[24,33,44]$. Our experimental data are consistent with the predicted half-metallic electronic structure. However, the expected full spin polarization appears to be in conflict with Andreev reflection spectroscopy results showing a spin polarization of $\sim 50 \%$, which is attributed to the scenario that one band is fully spin polarized while the other is unpolarized [45]. It has been argued that the half-metallic state can be tuned into an incomplete spin-polarized state by slight doping [34]. Further studies are required to resolve the disagreement.

Since Weyl points can only be defined in 3D momentum space, they will be removed by opening an energy gap 
under the 2D limit. If the band order is maintained during the crossover from 3D to 2D, the Weyl semimetal state can be transformed into the QAH insulator state. Band calculations have predicted that the QAH insulator state can be achieved under the 2D limit in $\mathrm{EuB}_{6}$, where the band crossings open an energy gap [24]. As there is no interference from other bands, the QAH insulator state has a global gap at $E_{F}$ in the density of states. These outstanding properties make $\mathrm{EuB}_{6}$ a promising system to study the physical behavior associated with a range of magnetic topological states in both 3D crystals and 2D thin films.

\section{ACKNOWLEDGMENTS}

T. Q. thanks Xi Dai for the initial idea. We thank Zhicheng Jiang, Zhenyu Yuan, Takayuki Muro, and Seigo Souma for technical assistance. This work was supported by the Ministry of Science and Technology of China (2016YFA0300600, 2019YFA0308602, 2016YFA0401000, 2017YFA0403401, and 2017YFA0302901), the National Natural Science Foundation of China (U1832202, 11874422, 11888101, U2032204, 12004416, 12074425, U1875192, and 11974395), the Chinese Academy of Sciences (QYZDBSSW-SLH043, XDB33020100, and XDB28000000), the Fundamental Research Funds for the Central Universities, and the Research Funds of Renmin University of China (19XNLG18 and 18XNLG14), the Beijing Municipal Science and Technology Commission
(Z171100002017018 and Z181100004218005), the Beijing Natural Science Foundation (Z180008), the K. C. Wong Education Foundation (GJTD-2018-01), the Center for Materials Genome, and the Users with Excellence Program of Hefei Science Center CAS (2019HSCUE001). N.P. thanks the Villum Fonden, for the NEED project (00027993), for support. M. S. was supported by the Sino-Swiss Science and Technology Cooperation (IZLCZ2-170075) and the Swiss National Science Foundation (200021_188413).

\section{APPENDIX A: TIME DEPENDENCE OF BAND DISPERSIONS MEASURED ON EU-TERMINATED SURFACES}

We study the changes of the electronic structures with time after cleavage on Eu-terminated surfaces, where two electron bands are observed near $E_{F}$ at $\bar{\Gamma}$ and $\bar{X}$, respectively. The bands shift monotonically downwards with time at a constant temperature $T=60 \mathrm{~K}$ (Fig. 5) and under temperature cycling (Fig. 6). We note that the results in Fig. 6 show that the FM transition has negligible effects on the band dispersions on Eu-terminated surfaces, which is significantly different from dramatic changes through the FM transition on B-terminated surfaces in Fig. 3(a). This behavior implies that the $4 f$ magnetic moments in the outmost layer on Eu-terminated surfaces may be disordered at low temperatures, which needs further study.

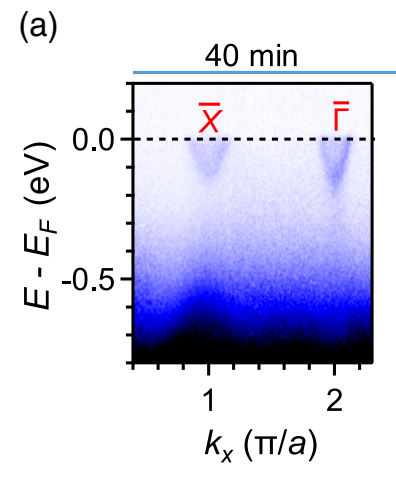

(b)

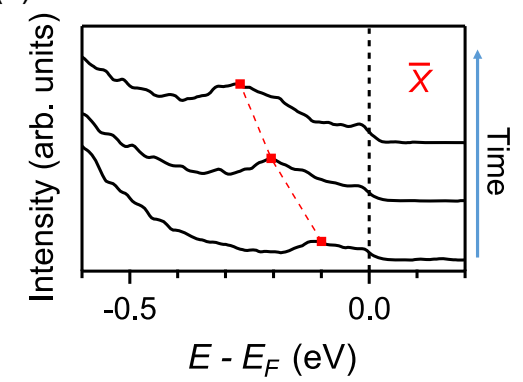

$140 \mathrm{~min}$

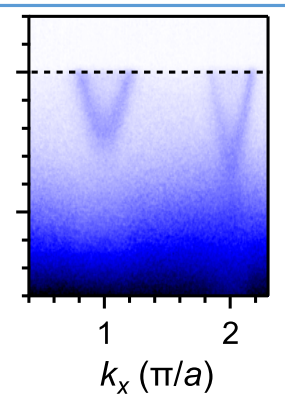

(c)

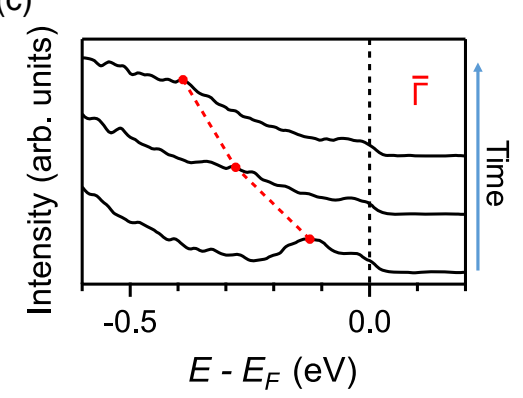

FIG. 5. Time dependence of band dispersions measured on Eu-terminated surfaces at $60 \mathrm{~K}$. (a) Intensity plots of the ARPES data collected at 40, 140, and 240 min after the cleavage with $h v=38 \mathrm{eV}$. (b),(c) Energy distribution curves at the $\bar{X}$ and $\bar{\Gamma}$ points, respectively, showing energy shifts with time of the band bottoms at $60 \mathrm{~K}$. 

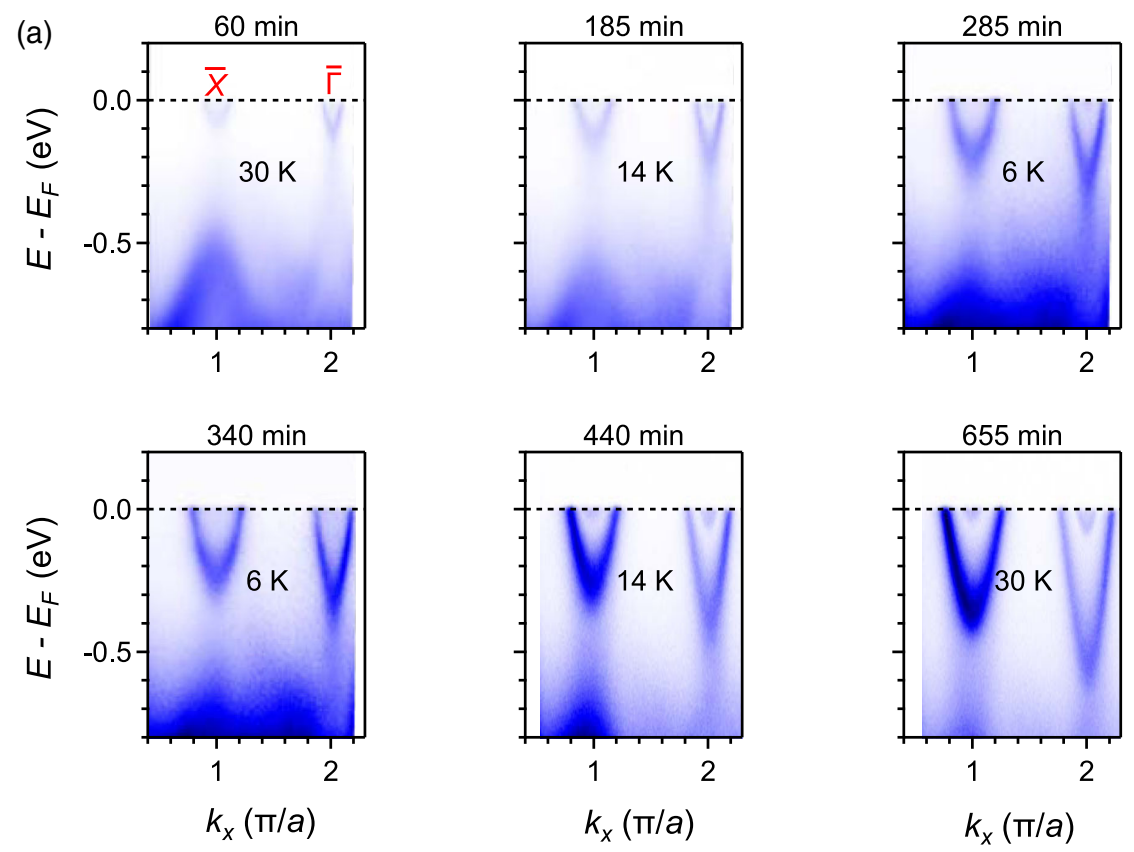

(b)

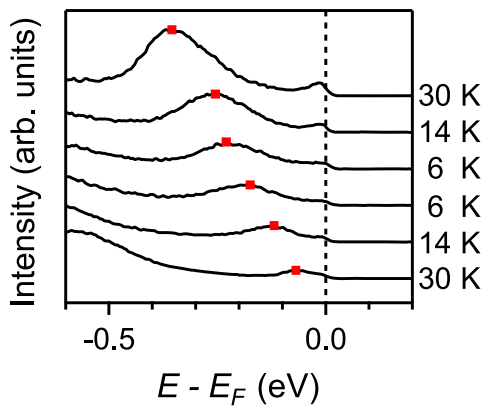

(c)

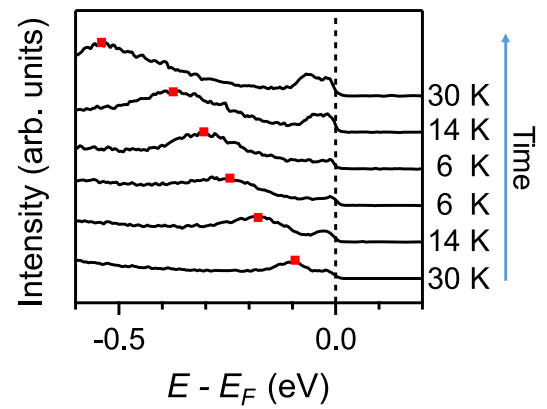

FIG. 6. Time dependence of band dispersions measured on Eu-terminated surfaces under temperature cycling. (a) Intensity plots of the ARPES data collected under temperature cycling in time sequence. (b),(c) Energy distribution curves at the $\bar{X}$ and $\bar{\Gamma}$ points, respectively, showing that energy shifts of the band bottoms are related to time rather than temperature.

\section{APPENDIX B: SURFACE DOPING EFFECTS ON EU- AND B-TERMINATED SURFACES}

Since the cleavage produces polar (001) surfaces with either Eu or B termination, it is expected that the electronic states on the polar surfaces suffer from surface doping. As $\mathrm{B}$ has a stronger electronegativity than $\mathrm{Eu}, \mathrm{Eu}$ has a positive valence state in $\mathrm{EuB}_{6}$. The surface doping would cause the electronic states on Eu-terminated surfaces to be more $n$ type compared with those in the bulk, which could explain the electron pockets observed on Eu-terminated surfaces in Figs. 2(d) and 2(e).

As $\mathrm{B}$ has a negative valence state in $\mathrm{EuB}_{6}$, the surface doping would cause the electronic states on B-terminated surfaces to be more $p$ type compared with those in the bulk. However, the electronic states observed on B-terminated surfaces appear to be either nearly neutral in Fig. 2(i) or slightly $n$ type in Fig. 7, which seems to contradict the expectation. One possible scenario is that the electronic states on B-terminated surfaces are coherent with the bulk states since they exhibit an obvious $k_{z}$ dispersion, which significantly reduces the surface doping effects. Another possibility is that the bulk states are slightly $n$ type because Hall effect measurements show a negative Hall coefficient [46-48], and the surface hole doping on B-terminated surfaces tends to neutralize the intrinsic $n$-type carriers.

The electronic states on B-terminated surfaces are observed to be nearly neutral in six samples and slightly $n$ type in two samples, indicating small fluctuations in the stoichiometry of the samples. In addition, the data in Fig. 7 confirm the semiconducting electronic structure with a narrow band gap in the PM state, which is consistent with the calculations in Fig. 1(d). 


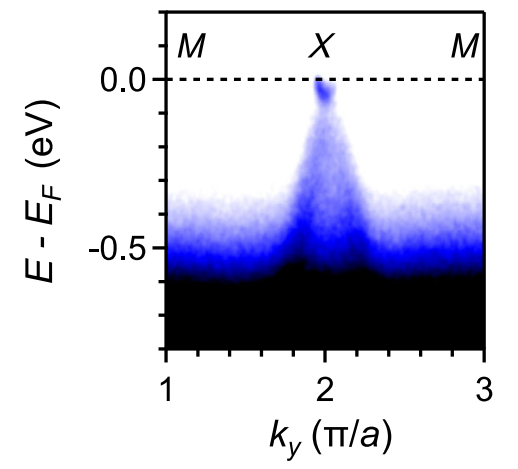

FIG. 7. Intensity plot of the ARPES data along $M-X$ in the $k_{x}-k_{y}$ plane with $k_{z}=8 \pi / a$ at $22 \mathrm{~K}$, showing slightly $n$-type electronic states on B-terminated surfaces of some samples.

[1] K. van Klitzing, G. Dorda, and M. Pepper, New Method for High-Accuracy Determination of the Fine-Structure Constant Based on Quantized Hall Resistance, Phys. Rev. Lett. 45, 494 (1980).

[2] D. J. Thouless, M. Kohmoto, M. P. Nightingale, and M. den Nijs, Quantized Hall Conductance in a Two-Dimensional Periodic Potential, Phys. Rev. Lett. 49, 405 (1982).

[3] F. D. M. Haldane, Model for a Quantum Hall Effect without Landau Levels: Condensed-Matter Realization of the "Parity Anomaly, Phys. Rev. Lett. 61, 2015 (1988).

[4] C. L. Kane and E. J. Mele, $Z_{2}$ Topological Order and the Quantum Spin Hall Effect, Phys. Rev. Lett. 95, 146802 (2005).

[5] B. A. Bernevig and S. C. Zhang, Quantum Spin Hall Effect. Phys. Rev. Lett. 96, 106802 (2006).

[6] B. A. Bernevig, T. L. Hughes, and S.-C. Zhang, Quantum Spin Hall Effect and Topological Phase Transition in HgTe Quantum Wells, Science 314, 1757 (2006).

[7] M. Konig, S. Wiedmann, C. Brune, A. Roth, H. Buhmann, L. W. Molenkamp, X.-L. Qi, and S.-C. Zhang, Quantum Spin Hall Insulator State in HgTe Quantum Wells, Science 318, 766 (2007).

[8] L. Fu and C. L. Kane, Topological Insulators with Inversion Symmetry, Phys. Rev. B 76, 045302 (2007).

[9] D. Hsieh, D. Qian, L. Wray, Y. Xia, Y. S. Hor, R. J. Cava, and M.Z. Hasan, A Topological Dirac Insulator in a Quantum Spin Hall Phase, Nature (London) 452, 970 (2008).

[10] R. Yuet al., Quantized Anomalous Hall Effect in Magnetic Topological Insulators, Science 329, 61 (2010).

[11] C.-Z. Chang et al. Experimental Observation of the Quantum Anomalous Hall Effect in a Magnetic Topological Insulator, Science 340, 167 (2013).

[12] J. Wang, B. Lian, and S.-C. Zhang, Quantum Anomalous Hall Effect in Magnetic Topological Insulators, Phys. Scr. T T164, 014003 (2015).

[13] J. G. Checkelsky, R. Yoshimi, A. Tsukazaki, K. S. Takahashi, Y. Kozuka, J. Falson, M. Kawasaki, and Y. Tokura, Trajectory of the Anomalous Hall Effect towards the
Quantized State in a Ferromagnetic Topological Insulator, Nat. Phys. 10, 731 (2014).

[14] X.-F. Kou et al., Scale-Invariant Quantum Anomalous Hall Effect in Magnetic Topological Insulators beyond the Two-Dimensional Limit, Phys. Rev. Lett. 113, 137201 (2014).

[15] C.-Z. Chang, W. Zhao, D. Y. Kim, H. Zhang, B. A. Assaf, D. Heiman, S.-C. Zhang, C. Liu, M. H. W. Chan, and J. S. Moodera, High-Precision Realization of Robust Quantum Anomalous Hall State in a Hard Ferromagnetic Topological Insulator, Nat. Mater. 14, 473 (2015).

[16] J.-H. Li, Y. Li, S. Du, Z. Wang, B.-L. Gu, S.-C. Zhang, K. He, W. Duan, and Y. Xu, Intrinsic Magnetic Topological Insulators in van der Walls Layered $\mathrm{MnBi}_{2} \mathrm{Te}_{4}$-Family Materials, Sci. Adv. 5, eaaw5685 (2019).

[17] Y.-J. Deng, Y. Yu, M. Z. Shi, Z. Guo, Z. Xu, J. Wang, X. H. Chen, and Y. Zhang, Quantum Anomalous Hall Effect in Intrinsic Magnetic Topological Insulator $\mathrm{MnBi}_{2} \mathrm{Te}_{4}$, Science 367, 895 (2020).

[18] C. Liu, Y. Wang, H. Li, Y. Wu, Y. Li, J. Li, K. He, Y. Xu, J. Zhang, and Y. Wang, Robust Axion Insulator and Chern Insulator Phases in a Two-Dimensional Antiferromagnetic Topological Insulator, Nat. Mater. 19, 522 (2020).

[19] J. Ge, Y.-Z. Liu, J.-H. Li, H. Li, T.-C. Luo, Y. Wu, Y. Xu, and J. Wang, High-Chern-Number and High-Temperature Quantum Hall Effect without Landau Levels, Natl. Sci. Rev. 7, 1280 (2020).

[20] L. Muechler, E. Liu, J. Gayles, Q. Xu, C. Felser, and Y. Sun, Emerging Chiral Edge States from the Confinement of a Magnetic Weyl Semimetal in $\mathrm{Co}_{3} \mathrm{Sn}_{2} \mathrm{~S}_{2}$, Phys. Rev. B 101, 115106 (2020).

[21] Q. Wang, Y. Xu, R. Lou, Z. Liu, M. Li, Y. Huang, D. Shen, H. Weng, S. Wang, and H. Lei, Large Intrinsic Anomalous Hall Effect in Half-Metallic Ferromagnetic $\mathrm{Co}_{3} \mathrm{Sn}_{2} \mathrm{~S}_{2}$ with Magnetic Weyl Fermions, Nat. Commun. 9, 3681 (2018).

[22] E.-K. Liu et al., Giant Anomalous Hall Effect in a Ferromagnetic Kagome-Lattice Semimetal, Nat. Phys. 14, 1125 (2018).

[23] D.-F. Liu et al. Magnetic Weyl Semimetal Phase in Kagome Crystal, Science 365, 1282 (2019).

[24] S. Nie, Y. Sun, F. B. Prinz, Z. Wang, H. Weng, Z. Fang, and X. Dai, Magnetic Semimetals and Quantized Anomalous Hall Effect in $\mathrm{EuB}_{6}$, Phys. Rev. Lett. 124, 076403 (2020).

[25] J. M. Tarascon, J. L. Soubeyroux, J. Etourneau, R. Georges, J. M. D. Coey, and O. Massenet, Magnetic Structures Determined by Neutron Diffraction in the $\mathrm{EuB}_{6-x} C_{x}$ System, Solid State Commun. 37, 133 (1981).

[26] Z. Fisk, D. C. Johnston, B. Cornut, S. von Molnar, S. Oseroff, and R. Calvo Magnetic, Transport, and Thermal Properties of Ferromagnetic $\mathrm{EuB}_{6}$, J. Appl. Phys. 50, 1911 (1979).

[27] T. Kasuya et al., Electronic Structure of $\mathrm{EuB}_{6}$, Transport and Magnetic Properties, J. Phys. (Paris), Colloq. 41, C5-161 (1980).

[28] J. M. Tarascon, J. Etourneau, P. Dordor, P. Hagenmuller, M. Kasaya, and J. M. D. Coey, Magnetic and Transport Properties of Pure and Carbon-Doped Divalent RE Hexaboride Single Crystal, J. Appl. Phys. 51, 574 (1980). 
[29] L. Degiorgi, E. Felder, H. R. Ott, J. L. Sarrao, and Z. Fisk, Low-Temperature Anomalies and Ferromagnetism of $\mathrm{EuB}_{6}$, Phys. Rev. Lett. 79, 5134 (1997).

[30] S. Süllow, I. Prasad, M. C. Aronson, J. L. Sarrao, Z. Fisk, D. Hristova, A. H. Lacerda, M. F. Hundley, A. Vigliante, and D. Gibbs, Structure and Magnetic Order of $\mathrm{EuB}_{6}$, Phys. Rev. B 57, 5860 (1998).

[31] M. L. Brooks, T. Lancaster, S. J. Blundell, W. Hayes, F. L. Pratt, and Z. Fisk, Magnetic Phase Separation in $\mathrm{EuB}_{6}$ Detected by Muon Spin Rotation, Phys. Rev. B 70, 020401 (R) (2004).

[32] C. S. Snow, S. L. Copper, D. P. Young, Z. Fisk, A. Comment, and J.P. Ansermet, Magnetic Polarons and the Metal-Semiconductor Transitions in $(\mathrm{Eu}, \mathrm{La}) B_{6}$ and EuO: Raman Scattering Studies, Phys. Rev. B 64, 174412 (2001).

[33] X.-H. Zhang, L. Yu, S. von Molnar, Z. Fisk, and P. Xiong, Nonlinear Hall Effect as a Signature of Electronic Phase Separation in the Semimetallic Ferromagnet $\mathrm{EuB}_{6}$, Phys. Rev. Lett. 103, 106602 (2009).

[34] J. H. Kim, W. Ku, C. C. Lee, D. S. Ellis, B. K. Cho, A. H. Said, Y. Shvydko, and Y. J. Kim, Spin-Split Conduction Band in $\mathrm{EuB}_{6}$ and Tuning of Half-Metallicity with External Stimuli, Phys. Rev. B 87, 155104 (2013).

[35] J. Kune and W. E. Pickett, Kondo and Anti-Kondo Coupling to Local Moments in $\mathrm{EuB}_{6}$, Phys. Rev. B 69, 165111 (2004).

[36] S. Wirth, S. Rößler, L. Jiao, M. V. A. Crivillero, P. F. S. Rosa, and Z. Fisk, Comparative Scanning Tunneling Microscopy Study on Hexaborides, Phys. Status Solidi B 258, 2000022 (2021).

[37] S. Rossler, L. Jiao, S. Seiro, P. F. S. Rosa, Z. Fisk, U. K. Rossler, and S. Wirth, Visualization of Localized Perturbations on a (001) Surface of the Ferromagnetic Semimetal $\mathrm{EuB}_{6}$, Phys. Rev. B 101, 235421 (2020).

[38] J. D. Denlinger, G.-H. Gweon, S.-K. Mo, J. W. Allen, J. L. Sarrao, A. D. Bianchi, and Z. Fisk, Absence of X-Point Band
Overlap in Divalent Hexaborides and Variability of the Surface Chemical Potential, J. Phys. Soc. Jpn. 71, 1 (2002).

[39] J. D. Denlinger, J. A. Clack, J. W. Allen, G. H. Gweon, D. M. Poirier, C. G. Olson, J. L. Sarrao, A. D. Bianchi, and Z. Fisk, Bulk Band Gaps in Divalent Hexaborides, Phys. Rev. Lett. 89, 157601 (2002).

[40] W. Henggeler, H.-R. Ott, D. P. Young, and Z. Fisk, Magnetic Ordering in $\mathrm{EuB}_{6}$, Investigated by Neutron Diffraction, Solid State Commun. 108, 929 (1998).

[41] R. G. Goodrich, N. Harrison, J. J. Vuillemin, A. Teklu, D. W. Hall, Z. Fisk, D. Young, and J. Sarrao, Fermi Surface of Ferromagnetic EuB 6 , Phys. Rev. B 58, 14896 (1998).

[42] M. C. Aronson, J. L. Sarrao, Z. Fisk, M. Whitton, and B. L. Brandt, Fermi Surface of the Ferromagnetic Semimetal, $\mathrm{EuB}_{6}$, Phys. Rev. B 59, 4720 (1999).

[43] I. Belopolski et al., Discovery of Topological Weyl Fermion Lines and Drumhead Surface States in a Room Temperature Magnet, Science 365, 1278 (2019).

[44] M. Kreissl and W. Nolting, Electronic Properties of $\mathrm{EuB}_{6}$ in the Ferromagnetic Regime: Half Metal versus Semiconductor, Phys. Rev. B 72, 245117 (2005).

[45] X.-H. Zhang, S. von Molnar, Z. Fisk, and P. Xiong, Spin-Dependent Electronic States of the Ferromagnetic Semimetal EuB 6 , Phys. Rev. Lett. 100, 167001 (2008).

[46] C. N. Guy, S. von Molnar, J. Etourneau, and Z. Fisk, Charge Transport and Pressure Dependence of $T_{c}$ of Single Crystal, Ferromagnetic $\mathrm{EuB}_{6}$, Solid State Commun. 33, 1055 (1980).

[47] S. Paschen, D. Pushin, M. Schlatter, P. Vonlanthen, H. R. Ott, D. P. Young, and Z. Fisk, Electronic Transport in $\mathrm{Eu}_{1-x} \mathrm{Ca}_{x} B_{6}$, Phys. Rev. B 61, 4174 (2000).

[48] V. V. Glushkov, A. V. Bogach, K. V. Gon'kov, S. V. Demishev, V. Yu. Ivanov, A. V. Kuznetsov, N. A. Samarin, N. Yu. Shitsevalova, K. Flachbart, and N. E. Sluchanko, Magnetic and Transport Properties of Colossal Magnetoresistance Compound $\mathrm{EuB}_{6}$, J. Exp. Theor. Phys. 105, 132 (2007). 